\title{
- Component Processes Subserving Rapid Automatized Naming in Dyslexic and Non-dyslexic Readers
}

\author{
Susana Araújo', Filomena Inácio', Ana Francisco', Luís Faísca', \\ Karl Magnus Petersson ${ }^{1,2,3,4}$ and Alexandra Reis ${ }^{1,2 *}$ \\ 'Cognitive Neuroscience Research Group, Institute for Biotechnology and Bioengineering, Centre for \\ Molecular and Structural Biomedicine, University of Algarve, Faro, Portugal \\ ${ }^{2}$ Cognitive Neurophysiology Research Group, Stockholm Brain Institute, Karolinska Institutet, Stockholm, \\ Sweden \\ ${ }^{3}$ Max Planck Institute for Psycholinguistics, Nijmegen, The Netherlands \\ ${ }^{4}$ Donders Institute for Brain, Cognition and Behaviour, Radboud University Nijmegen, Nijmegen, The \\ Netherlands
}

\begin{abstract}
The current study investigated which time components of rapid automatized naming (RAN) predict group differences between dyslexic and non-dyslexic readers (matched for age and reading level), and how these components relate to different reading measures. Subjects performed two RAN tasks (letters and objects), and data were analyzed through a response time analysis. Our results demonstrated that impaired RAN performance in dyslexic readers mainly stem from enhanced inter-item pause times and not from difficulties at the level of post-access motor production (expressed as articulation rates). Moreover, inter-item pause times account for a significant proportion of variance in reading ability in addition to the effect of phonological awareness in the dyslexic group. This suggests that non-phonological factors may lie at the root of the association between RAN inter-item pauses and reading ability. In normal readers, RAN performance was associated with reading ability only at early ages (i.e. in the reading-matched controls), and again it was the RAN inter-item pause times that explain the association. Copyright (C) 20II John Wiley \& Sons, Ltd.
\end{abstract}

Keywords: dyslexia; rapid naming; orthographic processing; response time analysis

\section{INTRODUCTION}

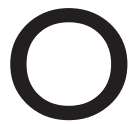

ne of the most robust concurrent and longitudinal predictors of reading outcome is rapid automatized naming (RAN; for a review, see Kirby, Roth, Desrochers, \& Lai, 2008). RAN performance is quantified in terms of how quickly children can name a visually presented array of items, typically high-frequency items (Denckla \& Rudel, 1976). Slow RAN is known to be associated with poor reading performance and to reliably distinguish dyslexic from non-dyslexic readers (e.g. Jones, Branigan, \& Kelly, 2009; for an overview, see Wolf, Bowers, \& Biddle, 2000). The relative contribution of RAN performance to reading scores is also stronger in less proficient

\footnotetext{
*Correspondence to: Alexandra Reis, Departamento de Psicologia, Faculdade de Ciências Humanas e Sociais, Campus de Gambelas, Universidade do Algarve, 8005-139 Faro, Portugal. E-mail: aireis@ualg.pt
} 
readers (Araújo, Pacheco, Faísca, Petersson, \& Reis, 2010; Johnston \& Kirby, 2006; McBride-Chang \& Manis, 1996).

Despite the acknowledged importance of RAN in predicting reading skills, the exact nature of this relation is not well-understood. Various researchers have suggested that RAN primarily reflects the access and retrieval of phonological codes from long-term memory (e.g. Chiappe, Stringer, Siegel, \& Stanovich, 2002; Pennington, Cardoso-Martins, Green, \& Lefly, 200I; Schatschneider, Carlson, Francis, Foorman, \& Fletcher, 2002; Torgesen, Wagner, \& Rashotte, 1994; Wagner, Torgesen, Laughon, Simmons, \& Rashotte, 1993). Others, have hypothesized that the 'phonological deficits and the processes underlying naming speed are separable sources of reading dysfunction' (Wolf \& Bowers, 1999, p.4I6), which suggests that there are independent processes involved in phonological and RAN tasks. Although there is substantial evidence showing that RAN accounts for unique variance in reading, beyond the effect of other measures of phonological processing (e.g. Kirby, Parrila, \& Pfeiffer, 2003; Landerl \& Wimmer, 2008; Manis, Doi, \& Bhadha, 2000), research on the relationship between reading, RAN and phonological skills have yielded mixed results (e.g. Cardoso-Martins \& Pennington, 2004; Patel, Snowling, \& de Jong, 2004; Parrila, Kirby, \& McQuarrie, 2004; Pennington et al., 200I). In line with a 'second deficit', Bowers and colleagues proposed an orthographic, rather than phonological, basis for the relation between RAN and reading (Bowers \& Newby-Clark, 2002; Bowers \& Wolf, 1993; Wolf et al., 2000). This hypothesis has since received some empirical support (Georgiou, Parrila, Kirby, \& Stephenson, 2008; Roman, Kirby, Parrila, Wade-Woolley, \& Deacon, 2009), although others dispute this view (Moll, Fussenegger, Willburger, \& Landerl, 2009; Papadopoulos, Georgiou, \& Kendeou, 2009). Finally, Jones and colleagues argue that both phonological and visual processes influence rapid naming (Jones, Obregón, Kelly, \& Branigan, 2008; see also Jones, Branigan, Hatzidaki, \& Obregón, 2010).

One constraint on the current understanding of the relationship between RAN and reading might be related to the fact that two sources of variance confound the interpretation of RAN results: the time to articulate each item - articulation time - and the duration of pauses between items in sequenced articulations-inter-item pause (Neuhaus, Foorman, Francis, \& Carlson, 200I). With few exceptions, previous research has approached RAN as a unitary measure, by obtaining a single performance time for the entire test (RAN total time). Evidence suggests that articulation times and inter-item pause times are not reliably related (Cobbold, Passenger, \& Terrel, 2003; Georgiou, Parrila, \& Kirby, 2006) and that these components are both uniquely predictive of reading efficiency (Georgiou, Parrila, \& Kirby, 2009). For example, the work of Georgiou, Parrila, and Liao, (2008) showed that each RAN component accounted for additional unique variance in reading, beyond the contribution shared by articulation and inter-item pause times. These findings suggest that the cognitive processes that underpin the articulation and pause time components of RAN are separable. Moreover, the use of eye-tracking methodology has proved useful in understanding the nature of the constituent components in RAN (see, for example the work of Jones et al., 2008).

Most of the research on RAN components has been conducted on children at an early stage of reading development. Also, this research has provided conflicting results. Although the studies mostly agree that inter-item pauses are key to understand the mechanisms that drive the RAN-reading relationship (e.g. Georgiou et al., 2006; Lervåg \& Hulme, 2009; Neuhaus, Foorman et al., 200I), the role of articulation time is less clear. For example, Georgiou et al. $(2006,2008)$ found that inter-item pause time was significantly correlated with reading accuracy and fluency, whereas articulation time was only weakly correlated with the reading measures. Variability in RAN total time is mostly related 
to inter-item pauses variability rather than variability in articulation times (Cobbold et al., 2003; Neuhaus, Carlson, Jeng, Post, \& Swank, 200I). Neuhaus, Foorman, et al. (200 I) also suggested that the association between RAN inter-item pauses and reading depends on the nature of the stimulus material, being significant for letter naming, nonsignificant for object naming and inconsistent for number naming. However, in a larger sample of Grade I children, the inter-item pause times were significantly associated with reading skills for both RAN letters and RAN objects, whereas the articulation times were associated with reading only in the RAN letters task (Neuhaus \& Swank, 2002). For work on older children, some studies report that neither articulation duration nor inter-item pause time were unique predictors of reading skill, when age and phonological skills were controlled (Clarke, Hulme, \& Snowling, 2005). Others have found that both components explain unique variance in reading fluency (Georgiou et al., 2009).

Likewise, which components of RAN explain the different relationship between RAN and reading in normal and dyslexic readers is not well-understood. Comparing these populations is of interest because deficits in RAN tasks have been linked to the reading level in dyslexic readers (e.g. Araújo et al., 2010). In an exception, Anderson, Podwall, and Jaffe, (1984), in a small study without reading-matched controls, found that both articulation and inter-item pause time were significantly longer for dyslexic children than for controls, whereas no articulation differences between groups were found in Obregon's work (1994, cited by Wolf \& Bowers, 1999; see also Snyder \& Downey, 1995). In the current study, we sought to clarify how RAN components account for the slow performance observed in dyslexia and how these are related to individual reading status in a well-controlled design with dyslexics and normal readers matched in terms of age and reading level. The use of age-matched and reading-matched control groups also provided an opportunity to investigate whether the relationship between the RAN components and reading changes during reading development.

In summary, the natural follow-up question concerns the sub-processes behind the timing components of RAN. Some hypotheses to the nature of inter-item pauses have been advanced, including an automatization index of phonological code retrieval (Neuhaus, Foorman et al., 200I). Alternatively, other authors have argued that at the beginning of reading development, it is the phonological-processing ability that mediates the relationship between alphanumeric RAN inter-item pauses and reading, whereas later on interitem pauses reflect the ease of building up orthographic representations (Georgiou, Parrila et al., 2008). On the other hand, the articulation component represents the actual production of speech and may be more indicative of stimulus familiarity or retrieval of stored representations (de Jong \& Van der Leij, 1999; Hulme, Roodenrys, Brown, \& Mercer, 1995).

In the present study, we disentangle the different cognitive processes engaged in RAN by analyzing articulation times and inter-item pauses from an alphanumeric (letter based) and a non-alphanumeric (object based) task in dyslexic and non-dyslexic readers. Specifically, the objective of the current study was twofold: first, to clarify which RAN component explains the group differences observed in normal and dyslexic readers; secondly, to examine the impact of RAN components on reading ability and shed more light on the underlying processes that mediate this relation. For this purpose, in addition to measuring the overall RAN total time, two time measures were extracted from the RAN tasksarticulation and inter-item pause times-and groups compared using a response time analysis. We also used a regression-based approach to assess the relative importance of RAN components in predicting different reading measures, including high-frequency word reading (an index of orthographic knowledge) and pseudoword reading (an index of phonological 
decoding), whereas controlling for phonological awareness. Because some recent papers have suggested that speeded naming tasks are mainly phonological processing speed tasks (e.g. Vaessen, Gerretsen, \& Blomert, 2009), we included accuracy and speed measures for phonological awareness as indices of phonological processing accuracy and speed, respectively.

In line with Bowers et al. (1993), our first general prediction is that the RAN total time would explain more unique variance in tasks involving a greater amount of orthographic processing. We also expected a greater association between RAN letters and reading compared with RAN objects, as the former entails more orthographic information processing. Moreover, and more specifically, we hypothesized that: (I) the inter-item pauses would be the main differentiator between normal and dyslexic readers; (2) the inter-item pauses would be associated with reading measures to a greater degree than articulation times, especially for the dyslexic subjects; and (3) if inter-item pause time is reflecting a key component in the RAN-reading association, and RAN taps non-phonological processing linked to orthographic skill, then inter-item pauses (especially for letters) should account for a large proportion of unique variance in high-frequency word reading as opposed to pseudoword reading.

\section{MATERIALS AND METHODS}

\section{Participants}

Informed consent was obtained from all the parents of participants in compliance with the Helsinki Declaration. Twenty-nine Portuguese children that had been diagnosed with dyslexia ( 17 boys and 12 girls, mean age $[ \pm s t d]=9.4 \pm[1.7]$ years) were recruited through private clinics that specialize in caring for children with learning disabilities. The inclusion criteria for the dyslexic participants were as follows: normal-range intelligence measured by the Raven Colored Matrices (Raven, Court, \& Raven, 1998); reading abilities significantly below grade mean level; absence of neurological, emotional or attention problems. The individual reading achievement was assessed through the 3DM reading test (see the succeeding text), adapted for the Portuguese population from the Differential Diagnosis Dyslexia Battery (Blomert \& Vaessen, 2009). Test-retest reliability was high (.9l). Scores were converted into z-scores with reference to normative data, which was collected in a large-scale study with 820 Portuguese children in grades I-4 (Reis et al., 20I I). The z-scores for the 5th grade children were estimated through polynomial regression procedures (Van Breukelen \& Vlaeyen, 2005), using the amount of months of formal reading instruction as predictor. Only those subjects who had reading speed scores at least I.5 SD below the grade mean level of the normative sample were included in the dyslexic group. The dyslexic group was matched with two control groups: 29 age-matched controls (I8 boys and II girls, mean age $[ \pm \mathrm{std}]=9.5 \pm[\mathrm{I} .8]$ years) and 29 reading-matched controls (I 4 boys and I 5 girls, mean age $[ \pm s t d]=7.0 \pm[.9]$ years), which were classified by their teachers as average pupils. All controls had intelligence scores in the normal range (Raven Colored Matrices) and reading scores (3DM reading test) within or above the average. There were no statistical differences between groups regarding intelligence scores ( $p>.05$, both comparisons). Dyslexic readers strongly differed from the age-matched $(p<.00 \mathrm{I})$ but not from the readingmatched controls $(p=.64)$ on the 3DM reading scores. Dyslexics and age-matched controls differed significantly from the reading-matched control group in terms of age $(p<.001$ for both). 


\section{Stimuli and Procedures}

All tasks were selected from the Differential Diagnosis Dyslexia Battery (Blomert \& Vaessen, 2009) and adapted for use in the Portuguese population (Reis et al., 20I I). The tasks were displayed on a computer screen, using Presentation software (version II.0; http://nbs. neurobs.com/presentation).

\section{Reading Measure}

The 3DM reading test was composed of two lists of high-frequency words and pseudowords. Each list was composed of 75 stimuli distributed on five sheets (I5 stimuli per sheet) of increasing difficulty with respect to the number of syllables (2-4), syllabic structure (with and without consonant clusters), and phoneme-grapheme correspondence rules (regular and irregular). For each list, the children had $30 \mathrm{~s}$ to read aloud as many words as possible. Reading speed was computed as the number of correctly read words per second.

\section{Phonological Awareness}

Phonological awareness was tested using a phoneme deletion task. Forty-four pseudowords were created by manipulation of word length (monosyllabic and disyllabic), syllabic structure (with and without consonant clusters) and position of the phoneme to be deleted (beginning, middle or end). Subjects listened via headphones to a given stimulus followed by the specific phoneme to be deleted, and they were instructed to repeat the resulting pseudoword without that specified phoneme. The percentage of correct responses was computed and used as the main dependent measure (phonological accuracy). The task has an internal consistency of .94 for accuracy. Speed of phonological awareness was also calculated by averaging the response latencies between the presentation of the word and the oral response (only correct answers were analyzed). The examiner pressed a button as soon as the subject gave an answer, and response times were automatically computed (time between stimulus offset and the button press). Reliability coefficient of the speed scores was 96 .

\section{Rapid Naming Repetition}

A rapid naming repetition task with letters and objects was designed based on the classical paradigm by Denckla and Rudel (1976). Five different stimuli were selected for letters (a, $d$, $\mathrm{O}, \mathrm{P}$ and $\mathrm{t}$ ) and objects (shoe, bed, glass, apple and fork) subtests; both were visually presented in two blocks. Each block had three columns of five stimuli, and thus, each stimulus was repeated three times per block presentation (I5 items per block). Subjects were instructed to name the stimuli as quickly and accurately as possible. The number of correctly named items per second was used as a measure of rapid naming speed. For each subject and task a wave file was created. Before the RAN test started, we ensured that the children knew the items by asking them to name all of the letters and objects in a practice trial. The naming test had a reliability of .82 .

\section{Analysis of Sound Files}

The digital sound files containing the letter-naming and object-naming responses for each subject were analyzed. Response times from the two RAN subtests were separated into 
articulation time (corresponding to the mean from onset to offset of the stimulus vocalization) and inter-item pause duration (corresponding to the mean of the time intervals between the sequenced articulations). The digitally recorded responses were manually timed using sound editing software (CoolEdit 2000). All articulation errors were removed from the analysis, along with the preceding and succeeding inter-item pause time. The response time measures were not considered when there was a self-correction, a pronoun before the stimulus name (e.g. 'an apple' instead of just saying 'apple'), or an extraneous verbalization (e.g. coughs). Inter-rater agreement was obtained on a random sample of one third of the data measured by two independent judges $(r=.98$ for inter-item pause and $r=.97$ for articulation time).

\section{RESULTS}

The raw scores from the reading test, the RAN subtests and the phonological awareness task were converted into z-scores with reference to the normative sample (stratified by grade), to improve comparability of performance levels between groups and tasks. Descriptive statistics for all measures are shown in Table I. As expected, dyslexic readers performed significantly worse than both control groups in all tested measures $(p<.00 \mathrm{I}$ for all; Tukey HSD Test).

Overall, low error rates were found in both RAN letter and RAN object subtests (4\% and $3 \%$, respectively). Statistical group differences for RAN total time were first tested with repeated measure ANOVAs, including the RAN subtest (objects versus letters) as within-subject factor and group (dyslexics versus age-matched versus reading-matched controls) as between-subject factor. A main effect of group was observed $[F(2,84)=26.8$; $p<.00 \mathrm{I}$ ], regardless of the RAN subtest-dyslexics performed significantly slower than age-matched and reading-matched controls $(p<.00$ I for both comparisons; Tukey HSD test). Furthermore, both control groups did not differed statistically from each other $(p=.49)$. As expected, the main effect of the task was not significant $(p=.28)$.

Table I. Average performance on cognitive tasks for the three reading groups

\begin{tabular}{|c|c|c|c|c|c|c|}
\hline & \multicolumn{2}{|c|}{ Dyslexics } & \multicolumn{2}{|c|}{ AC group } & \multicolumn{2}{|c|}{ RC group } \\
\hline & Mean & SD & Mean & SD & Mean & SD \\
\hline RWR speed (correct items/s) & -1.76 & .66 & .52 & .90 & .04 & .64 \\
\hline PWR speed (correct items/s) ${ }^{a}$ & -1.75 & .68 & .81 & .83 & -.04 & .63 \\
\hline Phoneme deletion (accuracy) ${ }^{a}$ & -1.39 & .55 & .39 & .72 & .01 & .75 \\
\hline Phoneme deletion (speed) ${ }^{\mathrm{a}}$ & -.80 & 1.18 & .18 & .78 & -.29 & .97 \\
\hline RAN letters (items/s) ${ }^{\mathrm{a}}$ & -.88 & I.II & .54 & 1.02 & .09 & .63 \\
\hline Articulation times (ms) & 332.50 & 52.52 & 290.10 & 43.22 & 342.63 & 99.32 \\
\hline Inter-item pauses (ms) & 410.34 & 246.04 & 221.91 & 129.33 & 478.77 & 252.13 \\
\hline RAN Objects (items $/ \mathrm{s})^{\mathrm{a}}$ & -.99 & .84 & .47 & 1.04 & -.08 & .73 \\
\hline Articulation times (ms) & 609.11 & 95.69 & 542.34 & 86.80 & 623.82 & 107.18 \\
\hline Inter-item pauses (ms) & 411.36 & 210.78 & 226.15 & 111.91 & 377.61 & 135.99 \\
\hline
\end{tabular}

Note: RWR, real word reading; PWR, pseudoword reading; RAN, rapid automatized naming; AC, age-matched controls; RC, reading-matched controls.

a: Standardized z-scores. 


\section{Analysis of the Inter-item Pause and Articulation Time}

To test for differences between groups in both RAN components, subjects' raw scores were used. Descriptive statistics are presented in Table I. The data were analyzed using repeated measures ANOVAs with the RAN subtest (objects versus letters) and RAN component (articulation time versus inter-item pause time) as within-subject factors and group (dyslexics versus age-matched versus reading-matched controls) as a betweensubject factor. The results demonstrated that there is a significant interaction between group and RAN component $[F(2,83)=7.5 ; p=.00 \mathrm{I}]$, showing that dyslexics and readingmatched controls differed from age-matched controls on inter-item times $(p<.00 \mathrm{I}$ for both comparisons) but not on articulation times $(p=.60$ and $p=.37$, respectively). Dyslexics and reading-matched controls did not differ on any RAN component $(p>.05$ for both comparisons; Tukey HSD test). A significant three-way interaction was also observed $[F(2,83)=6.2 ; p=.003]$. Although age-matched controls showed less inter-item pause time than articulation time, regardless of the RAN subtest (letters or objects), dyslexics and reading-matched controls showed larger inter-item pause time (over articulation time) during letter naming (Figure I).

Because the experimental groups differed significantly in their response times, we reanalyzed the data following suggestions by Faust, Balota, Spieler, and Ferraro, (1999) to ensure that over-additive effects did not contribute to the interactions. When the data were standardized with reference to subjects' individual means, the same pattern resulted, indicating that the outlined interactions are not explained by a spurious scale effect.

\section{Relationship between RAN and Reading Measures}

To investigate the unique contributions of RAN total time, articulation time and inter-item pauses to reading ability, a set of hierarchical regression analyses with changing order of the predictors were conducted for each group. The two RAN subtests (letters and objects) were analyzed separately. The relations were estimated after controlling for age and intelligence scores (entered as a block at step I) and phonological awareness accuracy (step 2). Next, the RAN measures were entered at the third step in the regression equation as the explanatory variables (one at a time). We did this separately for high-frequency words and pseudowords as the dependent variables resulting in a total of six regression
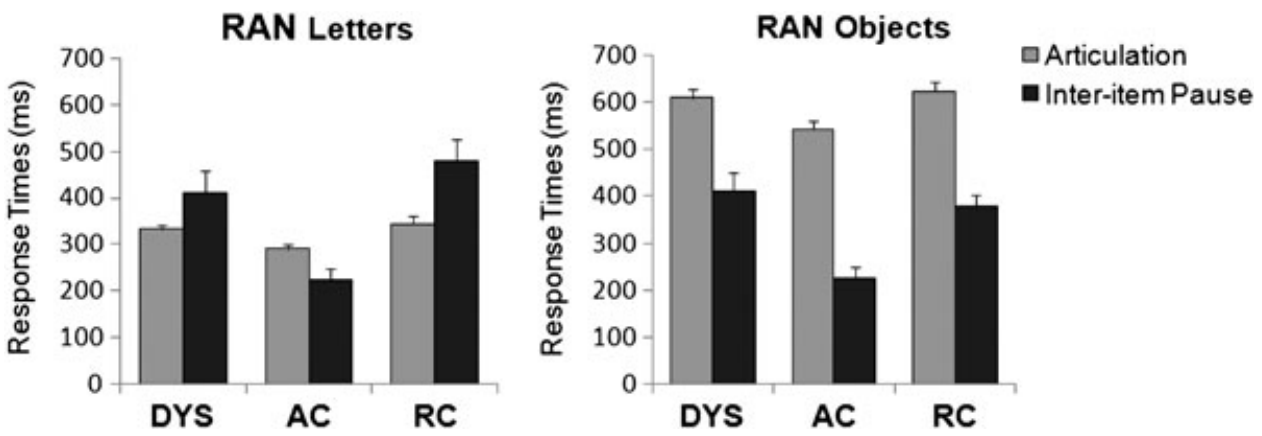

Figure I. Mean response times (ms) for rapid automatized naming (RAN) components, for the three reading groups (DYS, dyslexics; AC, age-matched controls; RC, reading-matched controls). The error bars represent standard error of the mean. 
analyses for each reading outcome. Using this procedure, we were able to investigate whether the components of RAN contributed significantly to reading measures when controlling for the other variables. The results for high-frequency word reading and pseudoword reading are summarized in Tables 2 and 3 , respectively. $R^{2}$ changes and the significance level are presented in both tables.

The results of the regression analyses revealed first that RAN total time (especially for letters) explained a unique variance of reading of high-frequency words and pseudowords in dyslexics (14.5\%-24.4\%) and in reading-matched controls (10.9\%-24.4\%), whereas its contribution in age-matched controls' reading fell to nonsignificant levels (Table 2 and 3).

At the time components level, data indicated that after age, intelligence level and phoneme deletion accuracy were entered into the equation only inter-item pause time continued to make a significant contribution to high-frequency word reading in dyslexics (letters, $\Delta R^{2}=15.1 \%$; objects, $\Delta R^{2}=12.2 \%$ ). None of the components of RAN

Table 2. Hierarchical regression analysis predicting high frequency word reading skills: Unique variance accounted by RAN response timing measurements

\begin{tabular}{|c|c|c|c|c|c|c|}
\hline & \multicolumn{2}{|c|}{ Dyslexics } & \multicolumn{2}{|c|}{ AC group } & \multicolumn{2}{|c|}{ RC group } \\
\hline & $\Delta R^{2}$ & $\Delta p$ & $\Delta R^{2}$ & $\Delta p$ & $\Delta R^{2}$ & $\Delta p$ \\
\hline I. Age + IQ & .015 & .820 & .135 & .162 & .049 & .517 \\
\hline $\begin{array}{l}\text { 2. Phoneme deletion } \\
\text { RAN letters }\end{array}$ & .201 & .018 & .023 & .429 & .268 & .004 \\
\hline 3. RAN total time & .244 & .003 & .060 & .197 & .109 & .043 \\
\hline 3. Inter-item pause & .151 & .025 & .046 & .260 & .162 & .012 \\
\hline $\begin{array}{l}\text { 3. Articulation time } \\
\text { RAN objects }\end{array}$ & .025 & .379 & .098 & .094 & .033 & .277 \\
\hline 3. RAN total time & .145 & .028 & .030 & .367 & .007 & .625 \\
\hline 3. Inter-item pause & .122 & .046 & .024 & .419 & .103 & .050 \\
\hline 3. Articulation time & .014 & .519 & .087 & .117 & .000 & .940 \\
\hline
\end{tabular}

Note: AC, age-matched controls; RC, reading-matched controls; RAN, rapid automatized naming.

Table 3. Hierarchical regression analysis predicting pseudoword reading skills: Unique variance accounted by RAN response timing measurements

\begin{tabular}{|c|c|c|c|c|c|c|}
\hline & \multicolumn{2}{|c|}{ Dyslexics } & \multicolumn{2}{|c|}{$A C$} & \multicolumn{2}{|c|}{$\mathrm{RC}$} \\
\hline & $\Delta R^{2}$ & $\Delta p$ & $\Delta R^{2}$ & $\Delta p$ & $\Delta R^{2}$ & $\Delta p$ \\
\hline I. Age + IQ & .098 & .263 & .024 & .740 & .027 & .696 \\
\hline $\begin{array}{l}\text { 2. Phoneme deletion } \\
\text { RAN letters }\end{array}$ & .224 & .008 & .267 & .006 & .201 & .017 \\
\hline 3. RAN total time & .211 & .003 & .001 & .837 & .244 & .003 \\
\hline 3. Inter-item pause & .060 & .140 & .010 & .58I & .277 & .001 \\
\hline $\begin{array}{l}\text { 3. Articulation time } \\
\text { RAN objects }\end{array}$ & .044 & .210 & .000 & .955 & .117 & .050 \\
\hline 3. RAN total time & .079 & .087 & .084 & .093 & .043 & .248 \\
\hline 3. Inter-item pause & .041 & .226 & .070 & .126 & .103 & .067 \\
\hline 3. Articulation time & .000 & .957 & .075 & .114 & .010 & .573 \\
\hline
\end{tabular}

Note: AC, age-matched controls; RC, reading-matched controls; RAN, rapid automatized naming. 
explained a significant amount of variance in dyslexics' pseudoword reading ability (albeit RAN total time for letters did, $\Delta R^{2}=21.1 \%$ ). In contrast, for age-matched controls, articulation time and inter-item pauses were always unrelated to measures of reading fluency (high-frequency word and pseudoword reading). For reading-matched controls, the relationship between RAN total time and high-frequency word reading fluency was mainly attributed to the inter-item pause time component, especially during letter naming (explained $10.3 \%-16.2 \%$ of the unique variance in high-frequency word reading). Both articulation time and inter-item pauses (RAN letters) accounted for considerable amount of unique variance in pseudoword reading fluency in this group (11.7\%-27.7\%), with interitem pauses' contribution being more substantial (Table 2 and 3 ).

At a final step, the regression analyses were repeated with age, intelligence scores, and both phonological awareness accuracy and speed entered before each component of RAN. Notably, the results were highly similar to all other analyses. Inter-item pauses continued to significantly predict dyslexics' reading of high-frequency words (letters, $\Delta R^{2}=$ 16.8\%; objects, $\Delta R^{2}=11.9 \%$ ). A unique contribution of inter-item pauses to high-frequency word reading (letters, $\Delta R^{2}=14.9 \%$ ) and to pseudoword reading (letters, $\Delta R^{2}=$ $28.5 \%$ ) was also observed for reading-matched controls.

\section{DISCUSSION}

The main objective of this study was to examine the relationship between RAN articulation and RAN inter-item pause time and reading proficiency in a sample of normal and dyslexic readers. Overall, the present study found that dyslexic readers are significantly slower on RAN tasks when compared with both age-matched and reading-matched controls. Both RAN letters and RAN objects were found to be different between groups, which suggest that the slow RAN performance in dyslexics is not due to a lower reading experience/ practice or a limited knowledge of letter names, and is therefore compatible with the idea that a failure to automatize the necessary skills for rapid naming reflects a core deficit in dyslexia (e.g. Wolf \& Bowers, 1999).

A more detailed analysis of the sources of naming variance indicated that slow RAN in dyslexics mainly stem from longer inter-item pauses and not articulate time. This finding extends previous research on unselected samples (Georgiou et al., 2006; Lervåg \& Hulme, 2009; Neuhaus, Foorman et al., 200 I) and suggests that the source of differences between normal and dyslexic readers is the efficiency of the cognitive process(es) that take place during inter-item pauses. Thus, the post-access articulatory-motor factors (reflected in articulation times) do not seem to be as important in distinguishing between reading groups. This outcome is inconsistent with early studies showing that dyslexics differed from normal readers both in terms of articulation duration and inter-item pause time (Anderson et al., 1984; Snyder \& Downey, 1995). Methodological differences may have accounted for this discrepancy. For instance, Anderson et al., (1984) used a small sample of dyslexics, thereby perhaps limiting the generality of the results.

The present study also offers some insight into how RAN total time, its components and reading ability are related and on the processes that may be responsible for this relationship. Consonant with previous studies (e.g. Araújo et al., 20 I0; Johnston \& Kirby, 2006), we found that the predictive strength of RAN to explain reading performance was dependent on age and reading level. Reading performance in dyslexics and readingmatched controls was found to be predicted by RAN total time, but no association was identified in age-matched controls. 
In addition, the regression analyses showed that dyslexics' reading performance was predicted by inter-item pause time but not articulation time, in both RAN tasks (letters and objects), compatible with recent reports (e.g. Lervåg \& Hulme, 2009). This highlights the role of RAN inter-item pauses in understanding factors related to slow reading. Similarly, the RAN inter-item pause component (especially for letters) seems to be at the root of the association between RAN and reading in normal readers at earlier ages (readingmatched controls). Thus, although RAN total time might be unreliably related to reading proficiency, RAN components, in particular inter-item pause, appears to be related to reading from the very start of reading acquisition. On the other hand, and contrary to the findings of Georgiou et al. (2009), we found no significant association between either of RAN components and reading proficiency in the age-matched controls, possibly because of an asymptotic level already attained by this sample in RAN and reading performance (McBride-Chang \& Manis, 1996). The age-matched controls in this study were generally good readers, a fact that may have attenuated the relation.

At the same time, to the extent that dyslexics' reading ability was still explained by RAN inter-item pauses after phonological awareness (accuracy and speed) was controlled, the results are not easily accommodated by an unitary phonological explanation for the relation between inter-item pauses and reading (e.g. Neuhaus, Foorman et al., 200 I; Neuhaus \& Swank, 2002). If it was the case that inter-item pauses primarily index phonological abilities and knowledge, we would have expected that the association with reading should no longer be significant when inter-item pauses were introduced after phonological awareness scores. Accordingly, in the dyslexic group, we found that although the RAN inter-item pause times explained a considerable amount of variance in high-frequency word reading, its contribution was not significant for pseudoword reading. This result, again, suggests that underlying phonological processing is unlikely to be the only explanation of why inter-item pauses are related to reading. In this case, we should rather observe a strong relation with pseudoword reading, as this depends more strongly on a detailed phonological analysis of the stimuli. Therefore, because of the greater involvement of orthographic processing in high-frequency word reading and the greater dependency of phonological processing ability in pseudoword reading, our findings suggest that at least in part RAN is related to variation in orthographic skills, consistent with the view of Bowers and colleagues (e.g. Bowers \& Ishaik, 2003; Bowers \& Newby-Clark, 2002). Also, an inspection of Table I and 2 suggests that the amount of variance explained in reading proficiency by the RAN total time was greater for alphanumeric RAN (letters) than for non-alphanumeric RAN (objects). However, we note that because of lack of power, this comparison was not statistically significant, and therefore, conclusion based on these differences need to be interpreted cautiously.

Other results support this orthographic hypothesis, including those showing that RAN is a better predictor of individual differences in 'pure' orthographic tasks (e.g. orthographic choice tasks) and text fluency than in grapheme-phoneme decoding tasks (Wolf et al., 2002). Georgiou, Parrila and colleagues (2008), in an unselected sample of Grades I to 3 students, also confirmed that inter-stimulus pause times are more strongly related to orthographic knowledge than other measures, including phonological awareness (see also Georgiou et al., 2009). Interestingly, the association between RAN inter-item pause times and orthographic knowledge increased across the developmental span, whereas the concurrent correlations between inter-item pauses and phonological awareness declined across time (Georgiou, Parrila et al., 2008). The present data seem to be in line with this finding. We observed that the RAN inter-item pause times only predicted fluency in high-frequency word reading in dyslexics, whereas in the younger reading-matched 
controls ( 7 years of age), the RAN inter-item pause times were more strongly associated with pseudoword reading (letters, $\Delta R^{2}=27.7 \%$ ). Thus, it seems likely that RAN inter-item pause reflects an underlying process which develops across time and thus changes its association with reading during reading acquisition.

Finally, although current evidence appears to favour a link between inter-item pauses and orthographic processing, especially in dyslexics, alternative explanations are still possible. In this study, we found that although the inter-item pauses in letter naming explained a greater amount of variance in the reading proficiency than inter-item pauses in object naming in the dyslexic group, the latter still explained a significant amount of individual variability. This suggests that RAN inter-item pauses may involve another mechanism in addition to orthographic processing. Similar to orthographic whole-word recognition of the letter strings, the RAN objects must be recognized and, like access from orthographic to phonological word representations in lexical reading, RAN requires activation of phonological entries via instantiated visual recognition units (Hawelka, Gagl, \& Wimmer, 2010). It is possible that longer inter-item pauses might also reflect the subjects' inability to integrate visual pattern information with stored representations and potentially, the slow access to phonological codes from visual recognition units. Recently, Stainthorp and colleagues investigated the relation between visual processing and poor RAN performance. They found that slow RAN children have difficulty in visual feature discrimination (Stainthorp, Stuart, Powell, Quinlan, \& Garwood, 2010). This issue clearly needs further investigation before a straightforward conclusion can be drawn.

In summary, the results showed that the relationship between RAN components and reading ability is dependent on age as well as reading level. We provide evidence that the process(es) indexed by the RAN inter-item pause time constitute the main source of naming difficulties in dyslexia, whereas the articulation times was unrelated to measures of reading. The current results suggest that the association between RAN inter-item pauses and reading is mediated by factors related to, or subserving, orthographic skills and orthographic skill development rather than with phonological processing ability.

\section{ACKNOWLEDGEMENTS}

This work was supported by the grant SFRH/BD/28488/2006, PTDC/PSI/64920/2006, PTDC/PSI-PCO/II0734/2009, and IBB/CBME, LA, FEDER/POCI 20I0, from Fundação para a Ciência e Tecnologia and the Swedish Dyslexia Foundation. The authors also thank Paula Teles from Clínica da Dislexia and Ana Guerreiro from Clínica Alvo for their help to contact dyslexic participants.

\section{REFERENCES}

Anderson, S. W., Podwall, F. N., \& Jaffe, J. (1984). Timing analysis of coding and articulation processes in dyslexia. Annals of the New York Academy of Sciences, 433, 7I-86.

Araújo, S., Pacheco, A., Faísca, L., Petersson, K. M., \& Reis, A. (2010). Visual rapid naming and phonological abilities: Different subtypes in dyslexic children. International Journal of Psychology, 45 (6), 443-452.

Blomert, L., \& Vaessen, A. (2009). Differentiaal diagnostiek van dyslexie; cognitieve analyse van lezen en spellen (Dyslexia differential diagnosis; cognitive analysis of reading and spelling). Amsterdam: Boom test publishers B. V. 
Bowers, P. G., \& Ishaik, G. (2003). RAN's contribution to understanding reading disabilities. In S. Graham, H. Swanson \& K. R. L. Harris (Eds.), Handbook of learning disabilities (Pp. I40-I57). New York: Guilford.

Bowers, P. G., \& Newby-Clark, E. (2002). The role of naming speed within a model of reading acquisition. Reading and Writing: An Interdisciplinary Journal, 15, 109-126.

Bowers, P. G., \& Wolf, M. (1993). Theoretical links among naming speed, precise timing mechanisms and orthographic skill in dyslexia. Reading and Writing: An Interdisciplinary Journal, 5, 69-85.

Cardoso-Martins, C., \& Pennington, B. F. (2004). The relationship between phoneme awareness and rapid serial naming skills and literacy acquisition: The role of developmental period and reading ability. Scientific Studies of Reading, 8(I), 27-52.

Chiappe, P., Stringer, R., Siegel, L. S., \& Stanovich, K. E. (2002). Why the timing deficit hypothesis does not explain reading disability in adults. Reading and Writing, 15, 73-107.

Clarke, P., Hulme, C., \& Snowling, M. (2005). Individual differences in RAN and reading: A response timing analysis. Journal of Research in Reading, 28(2), 73-86.

Cobbold, S., Passenger, T., \& Terrel, C. (2003). Serial naming speed and the component elements of speech time and pause time: Relationships with the development of word-level reading in children aged four to five years. Journal of Research in Reading, 26, 165-176.

Denckla, M. B., \& Rudel, R. G. (1976). Rapid "automatized" naming (R.A.N.): Dyslexia differentiated from other learning disabilities. Neuropsychologia, 14, 47I-479.

Faust, M. E., Balota, D. A., Spieler, D. H., \& Ferraro, F. R. (1999). Individual differences in informationprocessing rate and amount: Implications for group differences in response latency. Psychological Bulletin, 125, 777-799.

Georgiou, G. K., Parrila, R., \& Kirby, J. (2006). Rapid naming speed components and early reading acquisition. Scientific Studies of Reading, I0(2), 199-220.

Georgiou, G. K., Parrila, R., \& Kirby, J. (2009). RAN components and reading development from grade 3 to grade 5: What underlies their relationship? Scientific Studies of Reading, 13(6), 508-534.

Georgiou, G. K., Parrila, R., Kirby, J. R., \& Stephenson, K. (2008). Rapid naming components and their relationship with phonological awareness, orthographic knowledge, speed of processing, and different reading outcomes. Scientific Studies of Reading, 12(4), 325-350.

Georgiou, G. K., Parrila, R., \& Liao, C. (2008). Rapid naming speed and reading across languages that vary in orthographic consistency. Reading and Writing, 2I, 885-903.

Hawelka, S., Gagl, B., \& Wimmer, H. (20I0). A dual-route perspective on eye movements of dyslexic readers. Cognition, II5, 367-379.

Hulme, C., Roodenrys, S., Brown, G. D. A., \& Mercer, R. (1995). The role of long term memory mechanisms in memory span. British Journal of Psychology, 86, 527-536.

Johnston, T. C., \& Kirby, J. R. (2006). The contribution of naming speed to the simple view of reading. Reading and Writing, 19, 339-36I.

Jones, M. W., Branigan, H. P., Hatzidaki, A., \& Obregón, M. (2010). Is the "naming" deficit in dyslexia a misnomer? Cognition, I /6, 56-70.

Jones, M. W., Branigan, H. P., \& Kelly, M. L. (2009). Dyslexic and nondyslexic reading fluency: Rapid automatized naming and the importance of continuous lists. Psychonomic Bulletin and Review, 16(3), 567-572.

Jones, M. W., Obregón, M., Kelly, M. L., \& Branigan, H. P. (2008). Elucidating the component processes involved in dyslexic and non-dyslexic reading fluency: An eye-tracking study. Cognition, 109, 389-407.

de Jong, P. F., \& Van der Leij, A. (1999). Specific contribution of phonological abilities to early reading acquisition: Results from a Dutch latent variable longitudinal study. Journal of Educational Psychology, 9I, 450-476.

Kirby, J. R., Parrila, R. K., \& Pfeiffer, S. L. (2003). Naming speed and phonological awareness as predictors of reading development. Journal of Educational Psychology, 95(3), 453-464.

Kirby, J. R., Roth, L., Desrochers, A., \& Lai, S. (2008). Longitudinal predictors of word reading development. Canadian Psychology, 49(2), I03-I I0. 
Landerl, K., \& Wimmer, H. (2008). Development of word reading fluency and spelling in a consistent orthography: An 8-year follow-up. Journal of Educational Psychology, 100(I), 150-161.

Lervåg, A., \& Hulme, C. (2009). Rapid automatized naming (RAN) taps a mechanism that places constraints on the development of early reading fluency. Psychological Science, 20(8), 1040-1048.

Manis, F. R., Doi, L. M., \& Bhadha, B. (2000). Naming speed, phonological awareness, and orthographic knowledge in second graders. Journal of Learning Disabilities, 33(4), 325-333.

McBride-Chang, C., \& Manis, F. R. (1996). Structural invariance in the associations of naming speed, phonological awareness, and verbal reasoning in good and poor readers: A test of the double deficit hypothesis. Reading and Writing: An Interdisciplinary Journal, 8, 323-339.

Moll, K., Fussenegger, B., Willburger, E., \& Landerl, K. (2009). RAN is not a measure of orthographic processing: Evidence from the asymmetric German orthography. Scientific Studies of Reading, I3(I), I-25.

Neuhaus, G., Carlson, C. D., Jeng, W. M., Post, Y., \& Swank, P. (200I). The reliability and validity of rapid automatized naming scoring software ratings for the determination of pause and articulation component durations. Educational and Psychological Measurement 6I (3), 490-504.

Neuhaus, G., Foorman, B. R., Francis, D. J., \& Carlson, C. D. (200I). Measures of information processing in rapid automatized naming (RAN) and their relation to reading. Journal of Experimental Child Psychology, 78, 359-373.

Neuhaus, G., \& Swank, P. (2002). Understanding the relations between RAN letter subtest components and word reading in first-grade students. Journal of Learning Disabilities, 35(2), I58-174.

Papadopoulos, T. C., Georgiou, G. K., \& Kendeou, P. (2009). Investigating the double-deficit hypothesis in Greek. Journal of Learning Disabilities, 42(6), 528-547.

Parrila, R., Kirby, J. R., \& McQuarrie, L. (2004). Articulation rate, naming speed, verbal short-term memory, and phonological awareness: Longitudinal predictors of early reading development? Scientific Studies of Reading, 8, 3-26.

Patel, T. K., Snowling, M. J., \& de Jong, P. F. (2004). A cross-linguistic comparison of children learning to read in English and Dutch. Journal of Educational Psychology, 96, 785-797.

Pennington, B. F., Cardoso-Martins, C., Green, P. A., \& Lefly, D. L. (200I). Comparing the phonological and double deficit hypotheses for developmental dyslexia. Reading and Writing: An Interdisciplinary Journal, 14, 707-755.

Raven, J. C., Court, J. H., \& Raven, J. (1998). Coloured progressive matrices (I I th ed.). Oxford, UK: Oxford Psychologist Press.

Reis, A., Castro, S. L., Inácio, F., Pacheco, A., Araújo, S., Santos, M., et al. (20II). Versão Portuguesa da Bateria 3DM para avaliação da leitura e da escrita [3DM Portuguese version to assess reading and spelling skills]. Manuscript in preparation.

Roman, A. A., Kirby, J. R., Parrila, R. K., Wade-Woolley, L., \& Deacon, S. H. (2009). Toward a comprehensive view of the skills involved in word reading in grades 4, 6, and 8. Journal of Experimental Child Psychology, 102(I), 96-113.

Schatschneider, C., Carlson, C. D., Francis, D. J., Foorman, B. R., \& Fletcher, J. M. (2002). Relationship of rapid automatized naming and phonological awareness in early reading development: Implications for the Double-Deficit hypothesis. Journal of Learning Disabilities, 35(3), 245-256.

Snyder, L. S., \& Downey, D. M. (1995). Serial rapid naming skills in children with reading disabilities. Annals of Dyslexia, 45, 3I-50.

Stainthorp, R., Stuart, M., Powell, D., Quinlan, P., \& Garwood, H. (2010). Visual processing deficits in children with slow RAN performance. Scientific Studies of Reading, I4(3), 266-292.

Torgesen, J. K., Wagner, R. K., \& Rashotte, C. A. (1994). Longitudinal studies of phonological processing and reading. Journal of Learning Disabilities, 27, 276-286.

Vaessen, A., Gerretsen, P., \& Blomert, L. (2009). Naming problems do not reflect a second independent core deficit in dyslexia: Double deficits explored. Journal of Experimental Child Psychology, 103(2), 202-221.

Van Breukelen, G. J. P., \& Vlaeyen, J. W. S. (2005). Norming clinical questionnaires with multiple regression: The pain cognition list. Psychological Assessment, 17(3), 336-344. 
Wagner, R. K., Torgesen, J. K., Laughon, P., Simmons, K., \& Rashotte, C. A. (1993). Development of young readers' phonological processing abilities. Journal of Educational Psychology, 85, 83-I03.

Wolf, M., \& Bowers, P. G. (1999). The double-deficit hypothesis for the developmental dyslexias. Journal of Educational Psychology, 9 I (3), 4I5-438.

Wolf, M., Bowers, P. G., \& Biddle, K. (2000). Naming-speed processes, timing, and reading: A conceptual review. Journal of Learning Disabilities, 33(4), 387-407.

Wolf, M., O’Rourke, A. G., Gidney, C., Lovett, M., Cirino, P., \& Morris, R. (2002). The second deficit: An investigation of the independence of phonological and naming-speed deficits in developmental dyslexia. Reading and Writing: An Interdisciplinary Journal, 15, 43-72. 